\title{
OSTEOCHONDRITIS OF THE SUPERIOR TIBIAL EPIPHYSIS
}

\author{
J. I. Boldero and (i. P. Mitchell, Oxford, Exgland \\ From the Nuffield Orthopaedic Centre, Oxford
}

So far as we are aware, osteochondritis of the superior tibial epiphysis has not previously been described. It is, therefore, of interest to report the radiographic changes which were found in the superior tibial epiphysis of a boy aged twelve years, and which appear similar to those seen in osteochondritis elsewhere.

\section{CASE REPORT}

When the patient was first seen he complained of pain in the right knee of three months duration. There was no history of injury or recent illness; physical examination and radiographs were negative. He was kept under observation as an out-patient for three months. He was then admitted to hospital on account of local swelling, slight increase in heat and tenderness over the lateral tibial condyle. Radiographs showed evidence of increased density of the tibial epiphysis (Fig. 1).

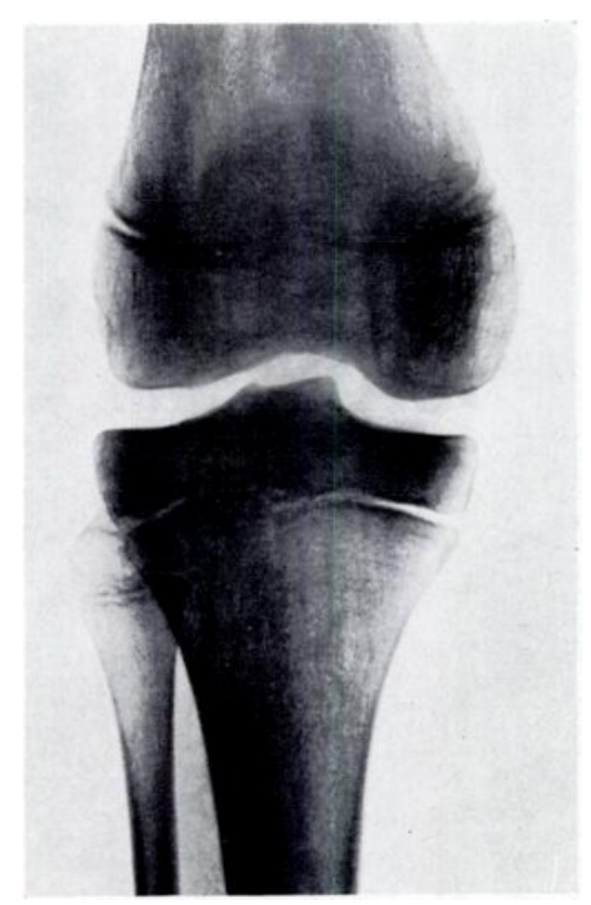

FIG. 1

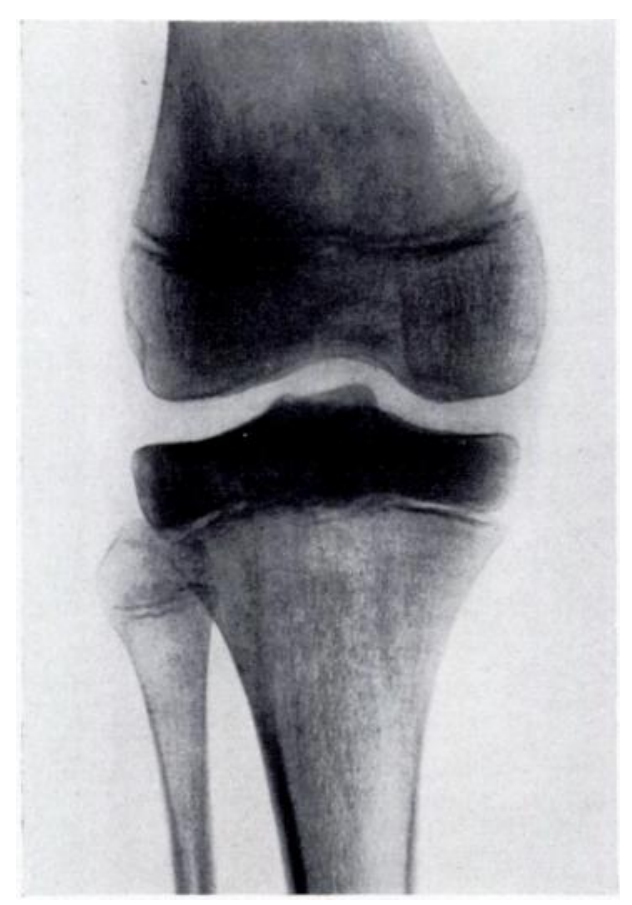

FIG. 2

Figure 1-Radiograph at time of admission, five months after the onset of symptoms. Figure 2-Seven months after onset.

During the next three months, investigation showed a consistently normal temperature and blood count. The Wassermann and Kahn reactions, agglutinations to salmonella and b. abortus and the Mantoux reaction were all negative. The blood sedimentation rate remained below 10 millimetres, apart from one rise to 25 millimetres. Radiographs two months after admission showed further increase in the epiphysial density (Fig. 2). This reached its 
greatest nine months after the onset (Fig. 3). Needle biopsy of the epiphysis showed a low-grade inflammatory reaction with no evidence of bone necrosis or absorption. An inguinal lymph gland removed at the same time was histologically normal.

Progress-Within a year of the first symptoms, the knee appeared quiescent. The radiograph showed advanced revascularisation (Fig. 4) and he was discharged in a non-weight-bearing

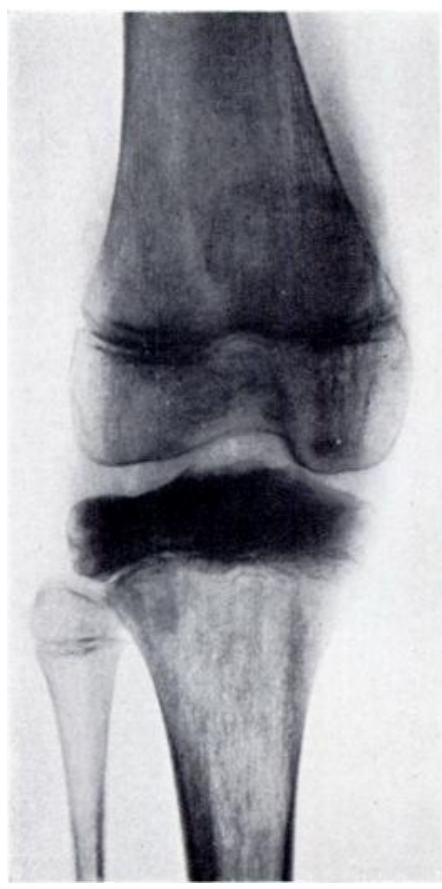

FIG. 3

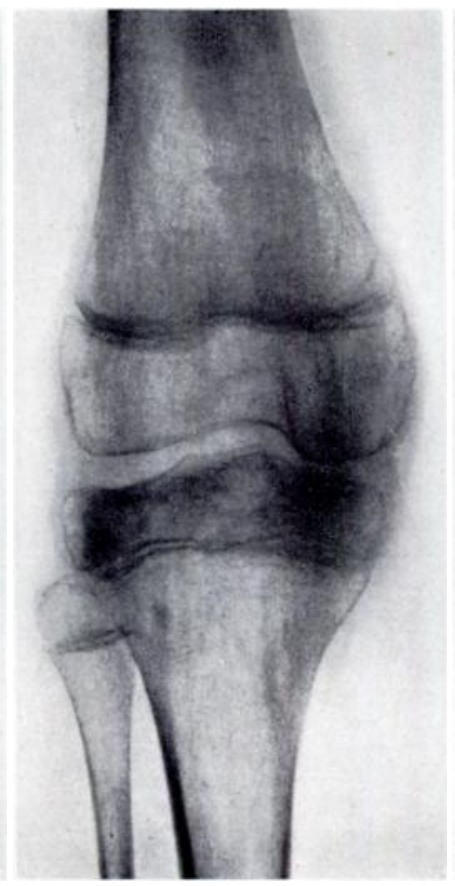

FIG. 4

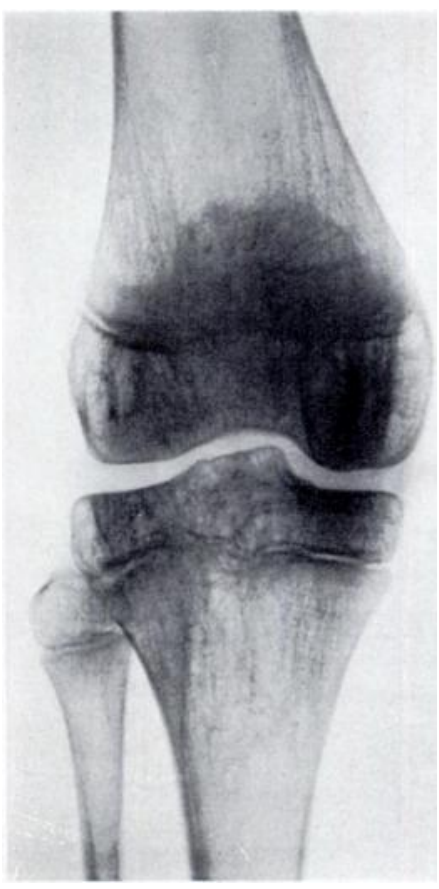

FIG. 5

Figure 3--Nine months after onset. Figure 4-Eleven months after onset. Figure 5 - Two and a half years after onset.

plaster. Subsequent progress was satisfactory; unprotected weight bearing was permitted a year and a half after onset of symptoms.

The patient was last seen two and a half years after the onset. He was indulging in normal activities without symptoms. Physical examination was negative, apart from slight wasting of the quadriceps. Radiographs showed a general improvement in the appearance of the bone (Fig. 5).

\section{DISCUSSION}

The diagnosis in this case lay between a pure ischaemic lesion and a low-grade infection in the epiphysis. Repeated blood counts and tests of sedimentation rate were normal except for one high reading of 25 millimetres in one hour. The temperature was consistently normal. No antibiotic treatment was given. The low-grade inflammatory reaction found on needle biopsy could be consistent with either lesion.

The clinical course and the radiographic findings of sclerosis followed by resorption and satisfactory reorganisation one year later suggest that this condition might be classed as an osteochondritis of the superior tibial epiphysis. 